\title{
Non-existence of weakly Pareto optimal allocations
}

\author{
Foivos Xanthos
}

Received: 28 April 2014 / Accepted: 3 May 2014 / Published online: 12 June 2014

(C) SAET 2014

\begin{abstract}
In this paper, we improve a characterization of the Riesz decomposition property, obtained in Aliprantis et al. (J Math Econ 92:55-76, 2000). As an application of this result, we show that the existence of weakly Pareto optimal allocations in some economic models is equivalent to the finite dimensional nature of the commodity space. This result enables us to give a characterization of infinite dimensional $C(K)$ spaces in terms of general equilibrium theory.
\end{abstract}

Keywords General equilibrium theory · Infinite dimensional commodity spaces · Pareto optimality $\cdot$ Riesz decomposition property

JEL Classification C62 $\cdot$ D51 $\cdot$ D61

\section{Introduction and mathematical preliminaries}

A challenging problem in general equilibrium theory is to extend equilibrium results of finite many commodities to the infinite dimensional setting. Several authors (Aliprantis and Brown 1983; Aliprantis et al. 2004; Aliprantis and Tourky 2009; Angelopoulos and Koutsougeras 2014; Bewley 1972; He and Yannelis 2014; Mas-Colell 1986; MasColell and Richard 1991; Podczeck 1996; Podczeck and Yannelis 2008; Yannelis and Prabhakar 1983; Yannelis 1985; Yannelis and Zame 1986) contributed to this problem and nowadays it is a well-known fact that such a generalization is possible, provided

This research was supported by NSERC.

F. Xanthos $(\varangle)$

Department of Mathematical and Statistical Sciences, University of Alberta,

Edmonton, AB T6G 2G1, Canada

e-mail: foivos@ualberta.ca 
that additional assumptions are imposed to the economic model. In Aliprantis et al. (1987); Araujo (1985); Jones (1984); Yannelis (1991), the authors demonstrated the necessity of additional assumptions by constructing concrete economies with infinitely many commodities, that fail to have an equilibrium or a non-empty core. In particular in Yannelis (1991), Example 6.2, the author illustrated that to ensure the non- emptiness of the core, it is not sufficient to assume that consumers' preferences have norm open lower sections. In Aliprantis et al. (1987) and Jones (1984), Example 8, are provided examples of economies in $C[0,1]$, where consumers' preferences are weakly continuous and the economy has an empty core. In Araujo (1985), the author showed that the same phenomenon occurs in $\ell_{\infty}$. In this paper, we throw light to these results, by showing that the existence of a weakly Pareto optimal allocation in these economies, forces the commodity space to be finite dimensional. In fact, we show that this is true for a general class of commodity spaces with an order unit. This phenomenon occurs due to the possible lack of compact intervals in infinite dimensional commodity spaces.

The existence of a Hausdorff linear topology under which the order intervals of the commodity space are compact is a standard assumption in economic models (see in Aliprantis and Tourky 2009; Podczeck and Yannelis 2008). In Sect. 2, we demonstrate that a large class of ordered vector spaces with the Riesz decomposition property, including the spaces $C[0,1], C^{1}[0,1], c$, fails to have compact intervals under any Hausdorff linear topology. This fact, enables us to improve a characterization of the Riesz decomposition property, obtained in Aliprantis et al. (2000). In general, the Riesz decomposition property of an ordered vector space $L$ cannot be characterized by the vector lattice property of the order dual $L^{\sim}$ (see Wickstead 2014, Example 10). In Aliprantis et al. (2000), Corollary 3.5, the authors showed that this is possible, if $L$ has an order unit, $\sigma\left(L, L^{\sim}\right)$-compact intervals and $L^{\sim}$ separates the points of $L$. In Theorem 2.5, we improve this result, by proving that in this case, $L$ is a finite dimensional vector lattice.

In Sect. 3, we study the existence of weakly Pareto optimal allocations in exchange economies. In our model, we assume that preferences are transitive, irreflexive, have non-empty, convex values and open lower sections with respect to some Hausdorff linear topology $\tau$. Under these assumptions, any economy on $L$ has an Edgeworth equilibrium, provided the set of allocations is $\tau$-compact (see Florenzano 2003, Proposition 5.2.2). In the case, where $L=C[0,1]$ and $\tau$ is the weak topology, we cannot always apply this result, since there are plenty of economies where the set of allocations is not weakly compact. It turns out that these economies fail to have a weakly Pareto optimal allocation. In particular, we show in Theorem 3.4, that for a general class of commodity spaces with an order unit, including any $C(K)$ space (see Corollary 3.5), the existence of a weakly Pareto optimal allocation for any economy is equivalent to the finite dimensional nature of the commodity space. This result gives a characterization of infinite dimensional spaces in terms of general equilibrium theory. In paper Polyrakis (2008), in a similar fashion, a characterization of reflexive Banach spaces, based on the existence of the demand correspondences is given.

In the case, where the commodity space is $\ell_{\infty}$, it is well known that the set of allocations is $w^{*}$-compact, so if we consider preferences that have $w^{*}$-open lower sections, then any economy will have a weakly Pareto optimal allocation. Hence, a natural guess would be that in the case of $C[0,1]$, the problem was the choice of the 
weak topology. As we show in Theorem 3.6, this is not the case, that is under any choice of Hausdorff linear topology $\tau$, there exists an economy in $C[0,1]$ with no weakly Pareto optimal allocations. This result, illustrates that when the commodity space fails to have compact intervals with respect to any Hausdorff linear topology, the validity of the Welfare theory in this model is questioned. To overcome this obstacle, we must impose additional conditions on the preferences. In the case, where the preferences are defined by utility functions, it is known that the compactness of the utility set can solve this problem (see Florenzano 2003, Remark 5.2.1 and Allouch and Florenzano 2004). To our knowledge, it is not known whether some additional condition can ensure the validity of the Welfare theory, in the general case where consumers' preferences are not defined by utility functions.

In this article, $L$ will denote an ordered vector space. The positive cone $L_{+}$of $L$ is assumed to be pointed (i.e. $\left.L_{+} \cap\left(-L_{+}\right)=\{0\}\right), L_{+}$defines a vector ordering on $L$, by letting $x \geq y$ whenever $x-y \in L_{+}$. We say that $L_{+}$is generating if $L=L_{+}-L_{+}$. The order interval $[x, y]$ is the set defined by $[x, y]=\{z \in L \mid x \leq z \leq y\}$. The space $L$ has the Riesz decomposition property, if for each $x, y, z \in L_{+}$such that $x \leq y+z$ there exist $x_{1} \in[0, y]$ and $x_{2} \in[0, z]$ such that $x=x_{1}+x_{2}$. If for every pair of finite non-empty subsets $A$ and $B$ of $L$ satisfying $A \leq B$ there exists $x \in L$ such that $A \leq x \leq B$, we say that $L$ has the interpolation property. It follows that the interpolation property and the Riesz decomposition property are equivalent (see Aliprantis and Tourky 2007, Theorem 1.54). If for every pair $x, y \in L$ their infimum $x \wedge y$ exists in $L$, we say that $L$ is a vector lattice or a Riesz space. We say that $L$ is $(\sigma-)$ monotone order complete if every increasing upper bounded (sequence) net of $L$ has a supremum. If $L$ is a vector lattice, this property is equivalent to the so-called $(\sigma-)$ Dedekind complete property, that is every upper bounded (countable) subset of $L$ has a supremum.

We denote with $L^{*}$, the algebraic dual of $L$ and $L^{\sim}$ the order dual of $L$. A functional $f \in L^{*}$ is said to be (strictly) positive, if $f(x) \geq 0(f(x)>0)$ for any $x \in L_{+} \backslash\{0\}$. If $L$ has the Riesz decomposition property and $L_{+}$is generating, then the order dual $L^{\sim}$ is a vector lattice (see Aliprantis and Tourky 2007, Theorem 1.61). We say that $e \in L_{+}$is an order unit of $L$, if $\bigcup_{n=1}^{\infty}[-n e, n e]=L$. An order unit $e$, induces the following seminorm on $L,\|x\|_{e}=\inf \{\lambda>0 \mid x \in[-\lambda e, \lambda e]\}$ (see Aliprantis and Tourky (2007), Theorem 2.55). For the following, assume that $L$ is equipped with a linear topology $\tau$. We denote with $L^{\prime}$ the topological dual of $L$ and we say that $L$ has compact (closed) intervals, whenever [0,x] is $\tau$-compact ( $\tau$-closed), for any $x \in L_{+}$. If $L$ has compact intervals then any order interval is $\tau$-compact, since whenever $x \leq y$ we have that $[x, y]=x+[0, y-x]$.

We recall here some well-known facts about Banach lattices and $C(K)$ spaces. A Banach lattice $L$ is a vector lattice, equipped with a complete norm such that $|x| \leq|y| \Rightarrow\|x\| \leq\|y\|$ for any $x, y \in L$. If $L$ is a Banach lattice, then $L^{\prime}=L^{\sim}$ (see Aliprantis and Border 2007, Theorem 9.11) and $e$ is an order unit of $L$ iff $e$ is an interior point of $L_{+}$(see Aliprantis and Tourky 2007, Theorem 2.8). An AM-space with a unit, is a Banach lattice with an order unit $e$ whose norm is given by $\|\cdot\|_{e}$. A Banach lattice is an AM-space with a unit if and only if is lattice isometric to $C(K)$ for some compact Hausdorff space $K$ (see Aliprantis and Burkinshaw 1985, Theorem 12.28). Assume for the following that $K$ is a compact Hausdorff space. The vector 
lattice $C(K)$ is $\left(\sigma^{-}\right)$Dedekind complete iff $K$ is $\left(\sigma^{-}\right)$Stonian, that is the closure of every open ( $F_{\sigma}$ ) set in $K$ is open (see Meyer-Nieberg 1991, Proposition 2.1.4, 2.1.5).

For unexplained mathematical terminology, we refer the reader to Aliprantis and Border (2007); Aliprantis and Burkinshaw (1985); Aliprantis and Tourky (2007).

\section{A characterization of the Riesz decomposition property}

In general, the Riesz decomposition property does not imply the vector lattice property. In the following result, we give a class of ordered vector spaces, in which this implication is possible.

Lemma 2.1 Suppose that L has the Riesz decomposition property and a generating cone. Then, $L$ is a vector lattice, if one of the following holds

(i) L is monotone order complete.

(ii) $L$ is $\sigma$-monotone order complete and $L$ has a strictly positive functional.

Proof For the following, suppose that $x, \tilde{x} \in L$ and $A=\{y \in L \mid y \leq x, \tilde{x}\}$ is the set of lower bounds of the set $\{x, \tilde{x}\}$. Note that the set $A$ is non-empty, since $L_{+}$is generating in $L$. Let $a, b \in A$, then by the interpolation property there exists $\gamma \in L$ such that $a, b \leq \gamma \leq x, \tilde{x}$, thus $\gamma \in A$. Therefore, the elements of $A$ can be viewed as an increasing net $\left(x_{a}\right)$ of $L$.

(i) Since $L$ is monotone order complete, we have that $w=\sup \left\{x_{\alpha}\right\} \leq x, \tilde{x}$ exists in $L$ and $w=x \wedge \tilde{x}$.

(ii) Let $f \in L^{*}$ be a strictly positive functional, then we can choose an increasing sequence $\left(x_{a_{n}}\right)$ such that $c=\sup \{f(x) \mid x \in A\}=\sup \left\{f\left(x_{a_{n}}\right) \mid n \in \mathbb{N}\right\}$. By the $\sigma$-monotone order completeness of $L$, we have that $w=\sup \left\{x_{a_{n}}\right\}$ exists in $L$. Note that $w \in A$ and by the choice of $\left(x_{a_{n}}\right)$, we have that $f(w)=c$. We claim that $w=x \wedge \tilde{x}$, indeed suppose that $z \leq x, \tilde{x}$, then by the interpolation property there exists $u \in L$ such that $z, w \leq u \leq x, \tilde{x}$, thus $u \in A$ and $f(u)=f(w)$. The strictly positivity of $f$, yields that $w=u \geq z$, hence $w=x \vee \tilde{x}$ and $L$ is a vector lattice.

We note here, that statement (ii) does not hold if we only assume that $L$ is $\sigma$ monotone order complete. We refer the reader to Goodearl (1986), Example 16.8 for an example of a $\sigma$-monotone order complete vector space with a generating cone, that has the Riesz decomposition property without being a vector lattice.

Proposition 2.2 If the order intervals are (countably) compact with respect to a Hausdorff linear topology $\tau$, then $L$ is a ( $\sigma-)$ monotone order complete vector space.

Proof Let $\left(x_{a}\right)_{a \in A}$ be an increasing net upper bounded by $x$. Fix some $a_{0} \in A$, then the net $\left(x_{a}\right)_{a \succeq a_{0}}$ is an increasing net in $\left[x_{a_{0}}, x\right]$. Since $\left[x_{a_{0}}, x\right]$ is compact, $\left(x_{a}\right)_{a \succeq a_{0}}$ contains a convergent subnet $\left(x_{a_{b}}\right)$. Let $z=\lim _{b} x_{a_{b}} \in\left[x_{a_{0}}, x\right]$, we will show that $z=\sup \left\{x_{a} \mid a \succeq a_{0}\right\}$. Fix some $a \succeq a_{0}$, then there exists $b_{0}$ such that $a_{b} \succeq a$ for each $b \succeq b_{0}$, thus $x_{a_{b}} \in\left[x_{a}, x\right]$ for each $b \succeq b_{0}$ and by taking the limit we have that $z \geq x_{a}$, since $\left[x_{a}, x\right]$ is closed. Assume that $w \geq x_{a} \geq x_{a_{0}}$ for each $a \succeq a_{0}$ then $z \leq w$, so 
$z=\sup \left\{x_{a} \mid a \succeq a_{0}\right\}$. Hence, we have that $z=\sup \left\{x_{a} \mid a \in A\right\}$. Indeed, let $a \in A$, then there exists $b \in A$ such that $b \succeq a, a_{0}$, thus $x_{a} \leq x_{b} \leq z$. For the countable case, the same proof works if we replace $A$ with $\mathbb{N}$ and use the fact, that every sequence in a countably compact set has a convergent subnet.

By applying Lemma 2.1 and Proposition 2.2, we have the following result. We note here that statement (i) is proved in Jameson (1970), 3.1.17 under the additional assumption that $L_{+}$is $\tau$ closed.

Corollary 2.3 Let L be an ordered vector space with the Riesz decomposition property and a generating cone. Assume that $L$ is equipped with a Hausdorff linear topology $\tau$, then $L$ is a vector lattice, if one of the following holds.

(i) The order intervals are compact.

(ii) The order intervals are countably compact and L has a strictly positive functional.

By the above, it follows that $C[0,1], c$ and $C^{1}[0,1]$ (see in Aliprantis and Border (2007), Example 8.5 and Theorem 16.11 and Aliprantis and Tourky (2007), Example 1.58) fail to have compact intervals with respect to any Hausdorff linear topology. In general, for $C(K)$ spaces, we have the following corollary.

Corollary 2.4 Let $K$ be a compact Hausdorff topological space that is not ( $\sigma$-) Stonian, then $C(K)$ fails to have (countable) compact intervals with respect to any Hausdorff linear topology.

The following result improves Corollary 3.5 in Aliprantis et al. (2000), where the authors proved the equivalence of (1) and (2).

Theorem 2.5 Assume that $L$ is an ordered vector space with an order unit and that $L^{\sim}$ separates the points in $L$. If the order intervals are $\sigma\left(L, L^{\sim}\right)$-compact, then the following statements are equivalent.

(1) $L^{\sim}$ is a vector lattice.

(2) L has the Riesz Decomposition Property.

(3) L is a finite dimensional vector lattice.

Proof (2) $\Rightarrow$ (3) We note here that $\sigma\left(L, L^{\sim}\right)$ is a Hausdorff linear topology, since $L^{\sim}$ separates the points of $L$. By Corollary 2.3 and Proposition 2.2, we have that $L$ is a Dedekind complete vector lattice. Let $e$ be an order unit of $L$. We consider the corresponding unit seminorm on $L,\|x\|_{e}=\inf \{\lambda>0 \mid x \in[-\lambda e, \lambda e]\}$ for each $x \in L$. Then, $\|\cdot\|_{e}$ is a norm on $L$, indeed suppose that $\|x\|_{e}=0$, then $x \in\left[-\frac{1}{n} e, \frac{1}{n} e\right]$ for each $n \in \mathbb{N}$, thus $\frac{1}{n} e \in[x, e]$ and $-\frac{1}{n} e \in[-e, x]$ for each $n \in \mathbb{N}$.

Since $\pm \frac{1}{n} e \stackrel{\sigma\left(E, E^{\sim}\right)}{\longrightarrow} 0$ and the order intervals are $\sigma\left(E, E^{\sim}\right)$-closed, we have that $x=0$. Note that $\left(L,\|\cdot\|_{e}\right)$ is a normed Riesz space, since $|x| \leq|y| \Rightarrow\|x\|_{e} \leq\|y\|_{e}$, for each $x, y \in L$. At the following, we will show that $\|\cdot\|_{e}$ is a Banach lattice. In view of Aliprantis and Border (2007), Theorem 9.3 it suffices to show that any increasing positive Cauchy sequence $\left(y_{n}\right)$ is convergent. Note that $\left(y_{n}\right)$ is an order bounded sequence, since it is $\|\cdot\|_{e}$-bounded, thus the Dedekind completeness of $L$ ensures 
that $a=\sup y_{n}$ exists in $L$. In particular, the above supremum coincides with the $\|\cdot\|_{e}$-limit. Indeed, let $\epsilon>0$ and pick $n_{0} \in \mathbb{N}$ such that $\left\|y_{n}-y_{n_{0}}\right\|_{e}<\epsilon$, for each $n \geq n_{0}$. Then, it follows that

$$
\begin{gathered}
y_{n}-y_{n_{0}} \leq \epsilon e \Rightarrow y_{n} \leq a \leq \epsilon e+y_{n_{0}} \Rightarrow \\
0 \leq a-y_{n} \leq \epsilon e \Rightarrow\left\|a-y_{n}\right\|_{e} \leq \epsilon \text { for all } n \geq n_{0}
\end{gathered}
$$

Therefore, $\left(L,\|\cdot\|_{e}\right)$ is an AM-space with a unit and by the Kakutani representation theorem it follows that $L$ is lattice isometrical isomorphic with a $C(K)$-space for some Hausdorff compact space $K$. Note that $L^{\sim}=L^{\prime}$ and the unit ball of $C(K)$ is the order interval $[-e, e]$, which is weakly compact by our assumptions. The later happens only if $K$ is a finite set (see Megginson 1998, Example 1.11.25). Hence, $L$ is a finite dimensional vector lattice.

\section{The economic model}

In this section, we consider a pure exchange economy

$$
\mathcal{E}=\left\langle(L, \tau),\left(P_{i}\right)_{i \in I},\left(\omega_{i}\right)_{i \in I}\right\rangle
$$

The set $I$ is finite and denotes the set of consumers. The ordered vector space $L$ represents the commodity space and it is assumed that it is equipped with a Hausdorff linear topology $\tau, L_{+}$is the consumption set of the consumers, $P_{i}: L_{+} \rightarrow L_{+}$is the preference of the $i$ consumer and $\omega_{i} \in L_{+}$is the initial endowment of the consumer $i$.

We recall here, that a correspondence $P: L_{+} \rightarrow L_{+}$is said to have $\tau$-open lower sections if the set $P^{-1}(x)=\left\{y \in L_{+} \mid x \in P(y)\right\}$ is relatively $\tau$-open, for any $x \in L_{+}$. We impose the following set of assumptions on consumer's preferences.

(A1) $P_{i}$ is irreflexive and transitive for each $i$.

(A2) $P_{i}$ has $\tau$-open lower sections.

(A3) $P_{i}$ has non-empty values.

(A4) $P_{i}$ has convex values.

Whenever we say that an economy $\mathcal{E}$ satisfies some of the following assumptions, we mean that these assumptions are satisfied by all the preferences of the economy.

Let $\omega=\sum_{i \in I} \omega_{i}$ be the total endowment. The set of (feasible) allocations is the following set.

$$
\mathcal{A}_{\omega}=\left\{\mathbf{x}=\left(x_{i}\right) \in\left(L_{+}\right)^{I} \mid \sum_{i \in I} x_{i} \leq \omega\right\} .
$$

Note that, whenever $L$ has compact intervals, the set of allocations is compact (with respect to the product topology).

Definition 3.1 An allocation $\left(x_{i}\right)$ is said to be weakly Pareto optimal if there is no allocation $\left(y_{i}\right)$ such that $y_{i} \in P_{i}\left(x_{i}\right)$ for each $i \in I$. 
The assumptions (A1) and (A2) are sufficient to yield the existence of a weakly Pareto optimal allocation for any economy $\mathcal{E}$, provided that the set of allocations is compact. This is a well- known result (see the corresponding proof in Florenzano (2003), Proposition 4.2.2) and we include here a proof for the sake of completeness.

Proposition 3.2 Any economy $\mathcal{E}$, such that $\mathcal{A}_{\omega}$ is compact and satisfies the assumptions $(A 1)-(A 2)$ has a weakly Pareto optimal allocation

Proof Let $P:\left(L_{+}\right)^{I} \rightarrow\left(L_{+}\right)^{I}$, the following correspondence $P\left(\left(x_{i}\right)\right)=$ $\prod_{i \in I} P_{i}\left(x_{i}\right)$, then $P$ is irreflexive, transitive and has open lower sections (with respect to the relative product topology). Suppose that $\mathcal{E}$ has no weakly Pareto optimal allocations, then we have that $\mathcal{A}_{\omega} \subseteq \bigcup_{\mathbf{x} \in \mathcal{A}_{\omega}} P^{-1}(\mathbf{x})$. Since $P^{-1}(\mathbf{x})$ is open and $\mathcal{A}_{\omega}$ is compact it follows that there exists a finite subset $F$ of $\mathcal{A}_{\omega}$ such that $\mathcal{A}_{\omega} \subseteq \bigcup_{\mathbf{x} \in F} P^{-1}(\mathbf{x})$. Hence, for any $\mathbf{x} \in F$, there exists some $\tilde{\mathbf{x}} \in F$ such that $\tilde{\mathbf{x}} \in P(\mathbf{x})$. Assume that $F$ has $n$ elements and choose some $\mathbf{x}_{1} \in F$, then since $P$ is irreflexive there exist $n-1$ possible $\tilde{\mathbf{x}} \in F$ such that $\tilde{\mathbf{x}} \in P\left(\mathbf{x}_{1}\right)$, let $\mathbf{x}_{2} \neq \mathbf{x}_{1}$ such that $\mathbf{x}_{2} \in P\left(\mathbf{x}_{1}\right)$. For $\mathbf{x}_{2}$, we have that $\mathbf{x}_{1} \notin P\left(\mathbf{x}_{2}\right)$, otherwise it follows by the transitivity of $P$ that $\mathbf{x}_{1} \in P\left(\mathbf{x}_{1}\right)$. Thus, there exist $n-2$ possible $\tilde{\mathbf{x}} \in F$ such that $\tilde{\mathbf{x}} \in P\left(\mathbf{x}_{2}\right)$, let $\mathbf{x}_{3} \neq \mathbf{x}_{1}, \mathbf{x}_{2}$ such that $\mathbf{x}_{3} \in P\left(\mathbf{x}_{2}\right)$. By repeating this argument, it follows that for the element $\mathbf{x}_{n}$ in this process, there is no element $\tilde{\mathbf{x}} \in F$ such that $\tilde{\mathbf{x}} \in P\left(\mathbf{x}_{n}\right)$, which is a contradiction.

Proposition 3.3 Let $X$ be a vector space, equipped with a topology $\tau$ and $K$ a closed subset of $X$. If $K$ has a countable open cover $\left\{U_{n}\right\}_{n \in \mathbb{N}}$ with no finite subcover, then there exists an irreflexive, transitive correspondence $\phi: X \rightarrow K$, that satisfies the following:

(i) $\phi(x)$ is non-empty for any $x \in X$,

(ii) $\phi$ has $\tau$-open lower sections.

If moreover $K$ and $U_{n}^{c}$ are convex for any $n$, then $\phi(x)$ is convex for any $x$.

Proof Let $\left\{U_{n}\right\}_{n \in \mathbb{N}}$ be a countable open cover of $K$ with no finite subcover. Put $U_{0}=K^{c}$ and for each $x \in X$, let $n(x)$ be the smallest $n \in \mathbb{N}$ such that $x \in \bigcup_{i=0}^{n} U_{i}$, then we define the following correspondence $\phi: X \rightarrow K$

$$
\phi(x)=\bigcap_{i=0}^{n(x)} U_{i}^{c} .
$$

Note that $\phi$ is transitive. Indeed, let $x, y, z \in X$ such that $y \in \phi(x)$ and $x \in \phi(z)$ then $y \notin \bigcup_{i=0}^{n(x)} U_{i}$, thus $n(x)<n(y)$ and $x \notin \bigcup_{i=0}^{n(z)} U_{i}$, thus $n(z)<n(x)$. Therefore, $n(z)<n(y) \Rightarrow y \notin \bigcup_{i=0}^{n(z)} U_{i} \Rightarrow y \in \phi(z)$. Also for each $x \in X$, we have that $x \notin \phi(x)$, since $x \in \bigcup_{i=0}^{n(x)} U_{i}$ and $\phi(x) \neq \emptyset$. Indeed, if $\bigcap_{i=0}^{n(x)} U_{i}^{c}=\emptyset$, then $K \subseteq$ $\bigcup_{i=1}^{n(x)} U_{i}$, which is a contradiction since the family $\left\{U_{n}\right\}_{n \in \mathbb{N}}$ has no finite subcover. If $K$ and $U_{n}^{c}$ are convex for any $n$, then $\phi(x)$ is convex for any $x$ as an intersection of convex sets. To verify assertion (ii), note that

$$
\phi^{-1}(x)=\bigcup_{i=0}^{n(x)-1} U_{i} \text { for each } x \in K
$$


Indeed, note that $y \in \phi^{-1}(x) \Leftrightarrow x \in \phi(y) \Leftrightarrow x \notin \bigcup_{i=0}^{n(y)} U_{i} \Leftrightarrow n(y)<n(x)$. Hence, $y \in \phi^{-1}(x) \Rightarrow y \in \bigcup_{i=0}^{n(y)} U_{i} \subseteq \bigcup_{i=0}^{n(x)-1} U_{i}$. Conversely, if $y \in \bigcup_{i=0}^{n(x)-1} U_{i}$, then $n(x)>n(y)$, thus $y \in \phi^{-1}(x)$.

We recall here that a topological space is said to be Lindelöf if every open cover has a countable subcover. A separable Banach space is Lindelöf with respect to the weak topology. In fact this is true for any weakly compact generated Banach space (see in Fabian et al. 2011, Theorem 14.31).

Theorem 3.4 Let $\left(L, \sigma\left(L, L^{\sim}\right)\right)$ be a commodity space, such that $L$ has $\sigma\left(L, L^{\sim}\right)$ closed intervals, an order unit, $L^{\sim}$ is a vector lattice and separates the points of $L$. If $\left(L, \sigma\left(L, L^{\sim}\right)\right)$ is a Lindelof space, then the following are equivalent.

(i) L is a finite dimensional space

(ii) Any economy $\mathcal{E}$ (with a single consumer) that satisfies assumptions (A1)-(A4) has a weakly Pareto optimal allocation.

Proof (i) $\Rightarrow$ (ii) If $L$ is a finite dimensional space, then it is easy to verify that $L_{+}$is closed and the order intervals are compact (see also Aliprantis and Tourky 2007, Lemma 3.1). Thus, the assertion follows by Proposition 3.2.

(ii) $\Rightarrow$ (i) Suppose that $L$ is not finite dimensional, then by Theorem 2.5 there exists $\omega \in L_{+}$such that $[0, \omega]$ is not $\sigma\left(L, L^{\sim}\right)$-compact. The following family

$$
\mathcal{S}=\left\{f^{-1}(-\infty, a), f^{-1}(b,+\infty), \mid a, b \in \mathbb{R}, f \in L^{\sim}\right\},
$$

is a subbase for $\sigma\left(L, L^{\sim}\right)$. By Alexander's subbase theorem, there exists an open cover $\left\{U_{i}\right\}_{i \in I}$ of $[0, \omega]$ with sets from $\mathcal{S}$ with no finite subcover, then $\left\{U_{i},[0, \omega]^{c}\right\}_{i \in I}$ is an open cover for $L$ and since $\left(L, \sigma\left(L, L^{\sim}\right)\right)$ is a Lindelöf space, there exists a countable subcover of $L$, thus we can find a countable open cover $\left\{U_{n}\right\}_{n \in \mathbb{N}}$ of $[0, \omega]$ with no finite subcover. All the assumptions of Proposition 3.3 for $X=L, K=[0, \omega]$ and $\tau=\sigma\left(L, L^{\sim}\right)$ are satisfied. Hence, we can define the single consumer economy $\mathcal{E}=\left\langle\left(L, \sigma\left(L, L^{\sim}\right)\right), P, \omega\right\rangle$, where $P$ is the correspondence of Proposition 3.3 restricted to $L_{+}$. Then, the economy satisfies assumptions (A1)-(A4) and has no weakly Pareto optimal allocation since for any $y \in[0, \omega]$ we have that $\emptyset \neq P(y) \subseteq[0, \omega]$.

Corollary 3.5 Let $(C(K), w)$ be a commodity space, where $K$ is a Hausdorff compact topological space and $w$ is the weak topology. Then, the following are equivalent.

(i) $C(K)$ is a finite dimensional space

(ii) Any economy $\mathcal{E}$ (with a single consumer) that satisfies assumptions (A1)-(A4) has a weakly Pareto optimal allocation.

Proof (i) $\Rightarrow$ (ii) Follows by Proposition 3.2.

(ii) $\Rightarrow$ (i) Suppose that $C(K)$ is infinite dimensional, then there exists a sequence $\left(x_{n}\right)$ in the unit ball of $C(K)$ with no convergent subsequence. Without loss of generality, we can assume that the constant function $\mathbf{1}$ is an element of this sequence. 
Let $M$ be the closed sublattice generated by $\left(x_{n}\right)$. Then, $M$ is a separable Banach lattice, with respect to the induced order $M_{+}=C(K)_{+} \cap M$ and norm (see in Kitover and Wickstead 2007, Proposition 2.1) . Note that $\mathbf{1}$ is an order unit of $M$ and that the $\sigma\left(M, M^{\prime}\right)$-topology coincides with the relative weak topology of $C(K)$. The assumptions of Theorem 3.4 are satisfied for the commodity space $\left(M, \sigma\left(M, M^{\prime}\right)\right)$, hence there exists an economy with a single consumer $\mathcal{E}=\left\langle\left(M, \sigma\left(M, M^{\prime}\right)\right), P, \omega\right\rangle$ which satisfies assumptions (A1)-(A4) and has no weakly Pareto optimal allocations. Next, we will extend this economy to the entire space. We define the following preference.

$$
\widetilde{P}(x)= \begin{cases}P(x) & \text { if } x \in M_{+} \\ M_{+} & \text {if } x \in C(K)_{+} \backslash M_{+}\end{cases}
$$

It is easy to see that the economy $\widetilde{\mathcal{E}}=\langle(C(K), w), \widetilde{P}, \omega\rangle$ satisfies the assumptions (A1), (A3), (A4). To see that (A2) holds, note that for any $x \in C(K)_{+} \backslash M_{+}$, we have that $\widetilde{P}^{-1}(x)=\varnothing$ and for any $x \in M_{+}$we have that $\widetilde{P}^{-1}(x)=\left(C(K)_{+} \backslash M_{+}\right) \cup$ $P^{-1}(x)$. Since $P: M_{+} \rightarrow M_{+}$has $\sigma\left(M, M^{\prime}\right)$-open lower sections, there exists a set $V$ that is $w$-open such that $P^{-1}(x)=V \cap M_{+}$, thus $\widetilde{P}^{-1}(x)=\left(C(K)_{+} \backslash M_{+}\right) \cup(V \cap$ $\left.C(K)_{+}\right)$is relatively $w$-open. By (ii), the economy $\widetilde{\mathcal{E}}$ has a weakly Pareto optimal allocation $\mathbf{x}=\left(x_{1}\right)$. Note that $x_{1} \in M_{+}$, since $\omega \in P(x)$ for any $x \in C(K)_{+} \backslash M_{+}$. Thus, $\mathbf{x}$ is also a weakly Pareto optimal allocation for $\mathcal{E}$, which is a contradiction.

Before we proceed to our final result, we note that by Corollary $2.4, C[0,1]$ and in general any $C(K)$ space, where $K$ is not $\sigma$-Stonian satisfies the assumptions of the following theorem.

Theorem 3.6 Let $(L, \tau)$ be a commodity space, such that $L$ is not $\sigma$-monotone order complete. Then, there exists an economy $\mathcal{E}$ with a single consumer that satisfies assumptions (A1)-(A3) and has no weakly Pareto optimal allocations.

Proof By Proposition 2.2, there exists an interval $[0, \omega]$ that is not countable $\tau$ compact, then there exists a countable cover of $[0, \omega]$ with no finite subcover. By applying Proposition 3.3, we can define the single consumer economy $\mathcal{E}=\langle(L, \tau)), P, \omega\rangle$ that satisfies assumptions (A1)-(A3) and $P: L_{+} \rightarrow[0, \omega]$. The economy $\mathcal{E}$ has no weakly Pareto optimal allocation since for any $y \in[0, \omega]$ we have that $\emptyset \neq P(y) \subseteq[0, \omega]$.

\section{References}

Aliprantis, C.D., Border, K.C.: Infinite dimensional analysis, 3rd Edition. Springer, Berlin (2007)

Aliprantis, C.D., Burkinshaw, O.: Positive operators. Springer, Berlin (1985)

Aliprantis, C.D., Tourky, R.: Cones and duality. Graduate Studies in Mathematics. American Mathematical Society, Providence, RI, vol. 84, pp. xiv+279 (2007). ISBN:978-0-8218-4146-4

Aliprantis, C.D., Brown, D.J.: Equilibria in markets with a Riesz space of commodities. J. Math. Econ 11(2), 189-207 (1983)

Aliprantis, C.D., Brown, D.J., Burkinshaw, O.: An economy with infinite dimensional commodity space and empty core. Econ. Lett 23, 1-4 (1987) 
Aliprantis, C.D., Tourky, R., Yannelis, N.C.: The Riesz Kantorovich formula and general equilibrium theory. J. Math. Econ 92, 55-76 (2000)

Aliprantis, C.D., Florenzano, M., Tourky, R.: General equilibrium analysis in ordered topological vector spaces. J. Math. Econ. 40(3-4), 247-269 (2004)

Aliprantis, C.D., Tourky, R.: Equilibria in incomplete assets economies with infinite dimensional spot markets. Econ. Theory 38(2), 221-262 (2009)

Allouch, N., Florenzano, M.: Edgeworth and Walras equilibria of an arbitrage-free exchange economy. Econ. Theory 23(2), 353-370 (2004)

Angelopoulos, A., Koutsougeras, L.C.: Value allocation under ambiguity. Econ. Theory (2014). doi:10.1007/s00199-014-0812-4

Araujo, A.: Lack of Pareto optimal allocations in economies with infinitelly many commodities: the need for impatience. Econometrica 53, 455-461 (1985)

Bewley, T.F.: Existence of equilibria in economies with infinitely many commodities. J. Econ. Theory 4(3), 514-540 (1972)

Fabian, M., Habala, P., Hajek, P., Montesinos, V., Zizler, V.: Banach space theory: The basis for linear and nonlinear analysis. Springer, New York (2011)

Florenzano, M.: General Equilibrium Analysis: Existence and Optimality Properties of Equilibria. Kluwer Academic Publishers, Boston, London (2003)

Goodearl, K.R.: Partially ordered abelian groups with interpolation, Mathematical Surveys and Monographs, 20. American MathematicalSociety, Providence, RI, pp. xxii+336 (1986). ISBN:0-8218-1520-2

He, W., Yannelis, N.C.: Equilibrium Theory Under Ambiguity. Mimeo (2014)

Jameson, G.: Ordered linear spaces. Springer, Berlin (1970)

Jones, L.E.: Special Problems Arising in the Study of Economies with Infinitely Many Com- modities, MEDS Discussion Paper No. 596, Northwestern Univesity (1984)

Kitover, A.K., Wickstead, A.W.: Invariant sublattices for positive operators. Indag. Math. (N.S.) 18(1), 39-60 (2007)

Mas-Colell, A.: The price equilibrium existence problem in topological vector lattices. Econometrica 54, 1039-1053 (1986)

Mas-Colell, A., Richard, S.F.: A new approach to the existence of equilibria in vector lattices. J. Econ. Theory 53, 1-11 (1991)

Megginson, R.E.: An introduction to Banach space theory. Springer, New York (1998)

Meyer-Nieberg, P.: Banach lattices, Universitext. Springer, Berlin (1991)

Podczeck, K.: Equilibria in vector lattices without ordered preferences or uniform properness. J. Math. Econ. 25, 465-484 (1996)

Podczeck, K., Yannelis, N.C.: Equilibrium theory with asymmetric information and with infinitely many commodities. J. Econ. Theory 141(1), 152-183 (2008)

Polyrakis, I.A.: Demand functions and reflexivity. J. Math. Anal. Apll. 338, 695-704 (2008)

Wickstead, A.W.: Polynomial Functions and the Riesz Interpolation Property. Indag. Math. (N.S.) 25, 395-404 (2014)

Yannelis, N. C.: The Core of an Economy without Ordered Preferences, in Equilibrium Theory in Infinite Dimensional Spaces. In: Khan, M. A., Yannelis, N.C. (eds.) Springer, Berlin (1991)

Yannelis, N.C., Prabhakar, N.D.: Existence of maximal elements and equilibria in linear topological spaces. J. Math. Econ. 12, 233-245 (1983)

Yannelis, N.C.: On a market equilibrium theorem with an infinite number of commodities. J. Math. Anal. Apll. 108, 595-599 (1985)

Yannelis, N.C., Zame, W.R.: Equilibria in Banach lattices without ordered preferences. J. Math. Econ. 15, 85-110 (1986) 